EPiC Series in Computing
Volume 75, 2021, Pages 59-68
$\begin{aligned} & \text { CAINE 2020. The 33rd International Conference on } \\ & \text { Computer Applications in Industry and Engineering }\end{aligned}$

\title{
On Designing Secured Communication Protocols along with Anonymity for CRT based Structured P2P Network Architecture
}

\author{
${ }^{1}$ Koushik Maddali, ${ }^{1}$ Swathi Kaluvakuri, ${ }^{2}$ Nick Rahimi, ${ }^{1}$ Bidyut Gupta and \\ ${ }^{3}$ Narayan Debnath \\ ${ }^{1}$ School of Computing \\ Southern Illinois University \\ Carbondale, IL, USA \\ ${ }^{2}$ Information Systems and Applied Technology \\ Southern Illinois University \\ Carbondale, IL, USA \\ ${ }^{3}$ School of Computing and Information Technology \\ Eastern International University, Vietnam
}

\{koushik, swathi.kaluvakuri, shrahimi \}@siu.edu, bidyut@cs.siu.edu, ndebnath@gmail.com

\begin{abstract}
In this work, we have considered designing secured communication protocols for Chinese remainder theorem based structured $\mathrm{p} 2 \mathrm{p}$ architecture. Such an architecture has been the choice because of the complexity in Inter or Intra group communications are just $O(1)$ [16]. In this work, we have considered efficient way to make the already existing communication protocols [16] secured. We have extended these protocols further to include anonymity. We have considered security separately for multicasting inside a group and multicasting outside the group.
\end{abstract}

Index Terms - Overlay multicast, Structured P2P network, Chinese Remainder Theorem, security, anonymity, cryptographic keys

\section{INTRODUCTION}

Problems associated with the global deployment of multicast-capable routers, lack of support for network management, and also the scalability problem caused by the simultaneous presence of large number of multicast sessions, are some of the main reasons why the deployment of router-based IP 
multicast has been slow. Consequently, researches have started considering application level multicast as an alternative to IP multicast, because the former one can be deployed fast as it does not depend on router infrastructure [1], [2], [3]. Multicast protocols proposed in [4], [5], [6] focus on designing an optimized overlay multicast tree per multicast source. They can work well for certain applications, such as software distribution. There exist some interesting multicast protocols designed to work in DHT-based architectures [7], [8] However, none of these above-mentioned works considers node heterogeneity. In addition, their performance degrades sharply as frequency of node (peer) movements in and out of the network increases. Note that frequent joining of new peers and leaving of existing peers is known as churn.

Authors in [9],[10], [11] have proposed a unique way of designing non-DHT based P2P network architecture. The architecture is an unrestricted ring of nodes (peers) in a sense that a node can be anywhere on the ring unlike in DHT-based ring, such as Chord [12]. Each node $\mathrm{n}_{\mathrm{i}}$ has its successor on the ring and also each node randomly selects $c_{i}$ number of other nodes on the ring as its immediate logical neighbors; $c_{i}$ is the degree/capacity of node $n_{i}$. They have proposed a capacity-constrained any source overlay multicast protocol which uses the unique idea of transforming a multicast problem to a broadcast one and the broadcast of a message is completed using a combination of tree propagation and sequential propagation on the ring. During multicasting, a tree structure is created implicitly even though there is no explicit multicast tree creation unlike in the classical multicast protocols that use either the sourcebased tree approach [13] or the shared tree approach [14].

The work [16] has used some of the ideas from [10] in designing efficient any source overlay p2p networks with capacity constrained approach.

\section{PRELIMINARIES}

In this section, we start with a brief description of the CRT-based hierarchical structured P2P architecture [9]; note that we design our multicast protocols for this architecture. This section ends with a summary of our contribution in the present work. This architecture is a two-level structured architecture for interest-based peer-to-peer system [9], [11], [15]. We use the following notations along with their interpretations while we define the architecture.

\section{Two Level Architecture}

A resource is defined as a tuple $\left\langle R_{i}, V>\right.$, where $R_{i}$ denotes the type of a resource and $V$ is the value of the resource. A resource can have many values. For example, let $R_{i}$ denote the resource type 'songs' and $\mathrm{V}^{\prime}$ denote a particular singer. Thus $\left\langle\mathrm{R}_{\mathrm{i}}, \mathrm{V}^{\prime}>\right.$ represents songs (some or all) sung by a particular singer $V^{\prime}$. In the proposed model for interest-based P2P systems, it is assumed that no two peers with the same resource type $R_{i}$ can have the same tuple; that is, two peers with the same resource type $R_{i}$ must have tuples $\left\langle\mathrm{R}_{\mathrm{i}}, \mathrm{V}^{\prime}>\right.$ and $\left\langle\mathrm{R}_{\mathrm{i}}, \mathrm{V}^{\prime \prime}>\right.$ such that $\mathrm{V}^{\prime} \neq \mathrm{V}^{\prime}$. However, this constraint can easily be relaxed [9].

We define the following. Let $S$ be the set of all peers in a peer-to-peer system. Then $S=\left\{P^{R i}\right\}, 0$ $\leq \mathrm{i} \leq \mathrm{n}-1$. Here $\mathrm{P}^{\mathrm{Ri}}$ denotes the subset consisting of all peers with the same resource type $\mathrm{R}_{\mathrm{i}}$ and no two peers in $P^{R i}$ have the same value for $R_{i}$ and the number of distinct resource types present in the system is n. Also, for each subset $\mathrm{P}^{\mathrm{Ri}}, \mathrm{P}_{\mathrm{i}}$ is the first peer among the peers in $\mathrm{P}^{\mathrm{Ri}}$ to join the system.

At level 1, there is a network of peers such that peers are directly connected (logically) to each other. In graph theoretic term, the network at level 1 is a complete graph. Hence, the network diameter is loverlay hop. The periphery of this network appears as a ring network and hence it is named as transit ring network. This network consists of the peers $\mathrm{P}_{\mathrm{i}}(0 \leq \mathrm{i} \leq \mathrm{n}-1)$. Therefore, number of peers on the ring is $\mathrm{n}$ and this number represents the number of distinct resource types present in the P2P system. Each of these $\mathrm{n}$ peers is termed group-head. The periphery of this network as well as the direct links connecting 
any two peers in this network can be used for efficient data lookup. In this architecture, each group-head has a global resource table (GRT) that has every group-head's logical as well as IP addresses.

At level-2, there are $\mathrm{n}$ numbers of completely connected networks of peers. Each such network, say $\mathrm{N}_{\mathrm{i}}$ is formed by the peers of the subset $\mathrm{P}^{\mathrm{Ri}},(0 \leq \mathrm{i} \leq \mathrm{n}-1)$, such that all peers $\left(\epsilon \mathrm{P}^{\mathrm{Ri}}\right)$ are directly connected (logically) to each other, resulting in the network diameter of 1 overlay hop. Each such $\mathrm{N}_{\mathrm{i}}$ also called group (in short as $\mathrm{G}_{\mathrm{i}}$ ) is connected to the transit ring network via the peer $\mathrm{P}_{\mathrm{i}}$, the group-head of $\mathrm{G}_{\mathrm{i}}$. The architecture is shown in Fig. 1.

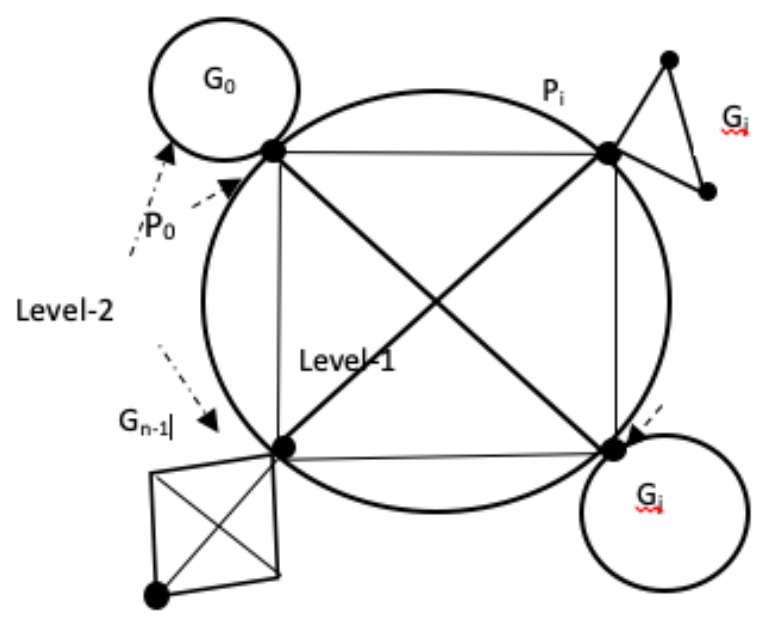

Fig. 1 A two-level structured P2P architecture with $r$ distinct resource types

In any structured P2P system, the mathematical model used to build the architecture defines neighborhood relations among peers. The mathematical model is intimately related to the efficiency of different data lookup schemes used in a given structured P2P system.

We now state a brief sketch of the mathematical model used in this approach to realize the architecture [9]. The authors first determine a simultaneous solution (a positive integer) of a given system of linear congruencies and then determine some more solutions as needed to form the architecture, which are congruent to the simultaneous solution. For this, the authors have used the Chinese Remainder Theorem (CRT). Each such solution will become the logical address of a group head uniquely [9] [16]. At the same time, it requires to determine separately the solutions of each linear congruence as needed and these solutions will represent the logical addresses of the peers present in a group [11]. The following interesting structural facts are revealed.

Observation 1. Any insertion of a group head $P_{l}$ always takes place between the current last group head $P_{l-1}$ and the first group head $P_{0}$ along the transit ring network.

Lemma 1. Diameter of the Level-1 network is 1 overlay hop.

Lemma 2. Diameter of a Level-2 group is 1 overlay hop.

Theorem 1. Diameter of the hierarchical two-level structured architecture is 3 overlay hops.

Remark 1. There are infinite number of solutions which are congruent to the one mutually congruent solution of any Linear Diophantine Equation (LDE) considered in CRT, hence, size of a cluster at Level2 can be made very large (theoretically unlimited), yet the diameter remains 1. 
Observation 2. Each group head has two different logical addresses; one from Level-1 assignment and one from Level 2 assignment.

Observation 3. Different group heads may get identical Level 2 assigned addresses. It will not affect any intra-cluster lookup query in a cluster, as this address is local to this group only.

\section{OUR CONTRIBUTION}

We have considered designing secured multicast protocols for both inside a group and for the two level architecture. In addition, we have also considered anonymity. We have also designed multicast algorithms with both security and anonymity. In section IV we present the secured data lookup algorithms. In section $\mathrm{V}$, we have present multicast algorithms with both security and anonymity.

\section{SECURED DATA LOOKUP ALGORITHMS}

Computer security encompasses confidentiality, integrity, availability, authentication, nonrepudiation etc. To achieve security in P2P networks from the view point of authentication and confidentiality, cryptographic algorithms are used. Generally, cryptographic algorithms are classified into two types, secret key cryptographic algorithms and public key cryptographic algorithms. Secret key cryptographic algorithms are also termed as symmetric key algorithms as the same key is used for both encryption and decryption which is shared between all parties involved. On the other hand, public key cryptographic algorithms are also known as asymmetric key algorithms. In this type, a pair of keys are used, one for encryption and another for decryption. One of the pair is made available to everyone known as a public key and the other is kept secret; known as the private key. Message encrypted using a public key can only be decrypted by the secret key of the pair. Same way, a message that is encrypted using a private key can only be decrypted by the public key of the pair. In this section, secure data lookup algorithms [9] both Inter and Intra are presented.

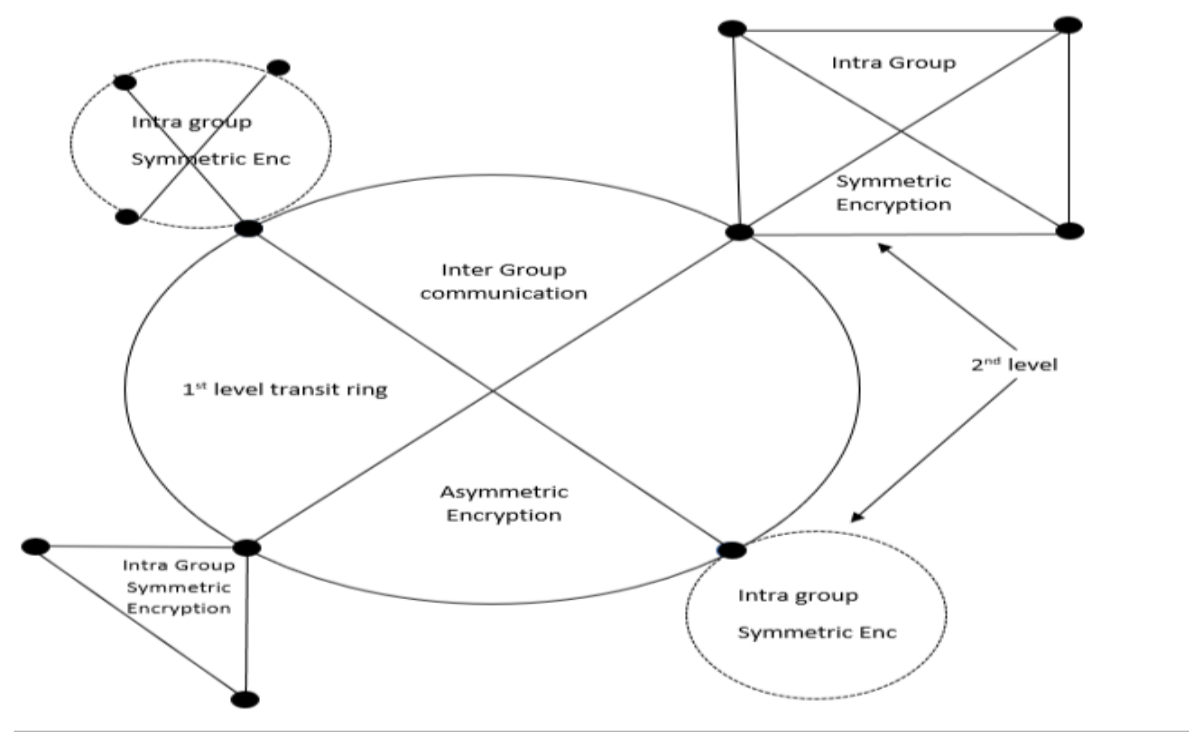

Fig. 2 Types of Cryptographic functions and their application domains 


\section{Secure Intra Group Lookup}

We use symmetric cryptography for secure intra group lookup. let us consider that in group Group , $_{i}$

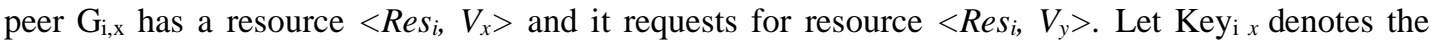
common key shared only by a peer $\mathrm{G}_{\mathrm{i}, \mathrm{x}}$ that belongs to Group ${ }_{i}$ and the corresponding group-head $H_{i}$ of the same group. To summarize, this common key information is known only to the peer $\mathrm{G}_{\mathrm{i}, \mathrm{x}}$ and group head $H_{i}$. So, symmetric encryption takes place with in a group.

1. Request node $\mathrm{G}_{\mathrm{i}, \mathrm{x}}$ encrypts the request $\left\langle\operatorname{Res}_{i}, V_{y}\right\rangle$ using the common key Key $\mathrm{i}_{x}$ and unicasts it to the Group head $H_{i}$

// This common key is known only to the requesting node and group head only so other nodes will not be able to decrypt it. Hence the symmetric key security

2. The Encrypted request is decrypted by Group head $H_{i}$ using the common key $\operatorname{Key}_{\mathrm{i} x}$

3. $H_{i}$ will broadcast the request $\left\langle\operatorname{Res}_{i}, V_{y}\right\rangle$ in Group $_{i}$,

4. If a node $\mathrm{G}_{\mathrm{i}, \mathrm{y}}$ has the requested resource $\left\langle\operatorname{Res}_{i}, V_{y}\right\rangle$

a. it encrypts the resource with common key $\mathrm{Key}_{\mathrm{i} y}$ and unicasts it to the group head $H_{i}$

b. The group head $H_{i}$ uses common key Key y and decrypts the response

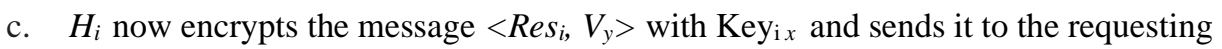
node $\mathrm{G}_{\mathrm{i}, \mathrm{x}}$

d. $\mathrm{G}_{\mathrm{i}, \mathrm{x}}$ decrypts the message using $\mathrm{Key}_{\mathrm{i} x}$ else

search for $\left\langle\operatorname{Res}_{i}, V_{y}\right\rangle$ fails

Fig. 3 Intra Group Lookup Algorithm with Security

\section{Secure Inter Group Lookup}

In this secured architectiure, any sort of communication between two peers $\mathrm{G}_{\mathrm{i}, \mathrm{x}} \in$ Group $_{i}$, and $\mathrm{G}_{\mathrm{j}, \mathrm{y}} \in$ Group $_{j}$ takes place only through the corresponsing group heads $H_{i}$ and $H_{j}$. Because the communication is between two different groups, we use asymmetric encryption, so here comes the concept of Public and Private keys.

We use the notations $P u b_{i}$ and $P r v_{i}$ to denote respectively the public and private keys of group-head $H_{i}$ and let $P u b_{j}$ and $\operatorname{Prv}_{j}$ be the public and private keys of group-head $H_{j}$. Without any loss of generality, let a peer $\mathrm{G}_{\mathrm{i}, \mathrm{x}} \in$ Group $_{i}$ requests for a resource $\left\langle\operatorname{Res}{ }_{j}, V_{y}\right\rangle$. Peer $\mathrm{G}_{\mathrm{i}, \mathrm{x}}$ is aware of the fact that that $\operatorname{Res}_{j} \notin$ Group $_{i}$. 
1. Request node $\mathrm{G}_{\mathrm{i}, \mathrm{x}}$ encrypts the request $\left\langle\operatorname{Res}_{\mathrm{j}}, V_{y}\right\rangle$ using the common key $\mathrm{Key}_{\mathrm{i} x}$ and unicasts it to the Group head $H_{i}$

// This common key is known only to the requesting node and group head only so other nodes will not be able to decrypt it.

2. The Encrypted request is decrypted by Group head $H_{i}$ using the common key $\mathrm{Key}_{\mathrm{i} x}$ and finds the Group head address of $H_{j}$ along with its public key Pub $b_{j}$ from the GRT table

3. $H_{j}$ encrypts the message with $P u b_{j}$ and forwards the request across the ring

4. Each intermediate group-head $H_{k}$ forwards the request until the request arrives at $H_{j}$

5. Now $H_{j}$ will decrypt the message using its private key $\operatorname{Pr} v_{j}$

6. if $H_{j}$ itself possesses $\left\langle\operatorname{Res}_{j}, V_{y}\right\rangle$

7. $\quad H_{j}$ encrypts the message with the public key $P u b_{i}$ of $H_{i}$ and unicasts it to $H_{i}$

8. else

9. $\quad H_{j}$ broadcasts the request for $\left\langle\operatorname{Res}_{j}, V_{y}\right\rangle$ in group Group $_{j}$

10. $\quad$ if $\exists G_{j, y}\left(\epsilon\right.$ Group $\left._{j}\right)$ which possesses $\left\langle\operatorname{Res}_{j}, V_{y}\right\rangle$

11. $G_{j, y}$ encrypts the request message with $\operatorname{Key}_{j y}$

12. $\quad H_{j}$ decrypts the message with $K e y_{j y}$

13. $H_{j}$ encrypts the decrypted message with the public key $P u b_{i}$ of $H_{i}$ and sends it to $H_{i}$

14. $\quad H_{i}$ decrypts the message with its own private key $\operatorname{Pr} v_{i}$

15. $H_{i}$ encrypts the message $\left\langle\operatorname{Res}_{j}, V_{y}\right\rangle$ with $K_{e} y_{i x}$ and sends it to the requesting peer $\mathrm{G}_{\mathrm{i}, \mathrm{x}}$

16. $\mathrm{G}_{\mathrm{i}, \mathrm{x}}$ decrypts the received message using the common key Key ${ }_{i x}$

$17 . \quad$ else

$18 . \quad H_{j}$ unicasts 'search failed message' to $H_{i}$

$19 . \quad$ end

20. End

Fig. 4 Inter Group Lookup Algorithm with Security

\section{SECURED MULTICAST ALGORITHMS WITH ANONYMITY}

In this section we'll present secured multicast algorithms both inter group and for the whole p2p systems. The basic Multicast algorithms of CRT architecture presented in [16] have been enhanced here with security properties.

Secured Multicast Algorithm 1 with Anonymity where capacity $\geq$ \#groupheads 


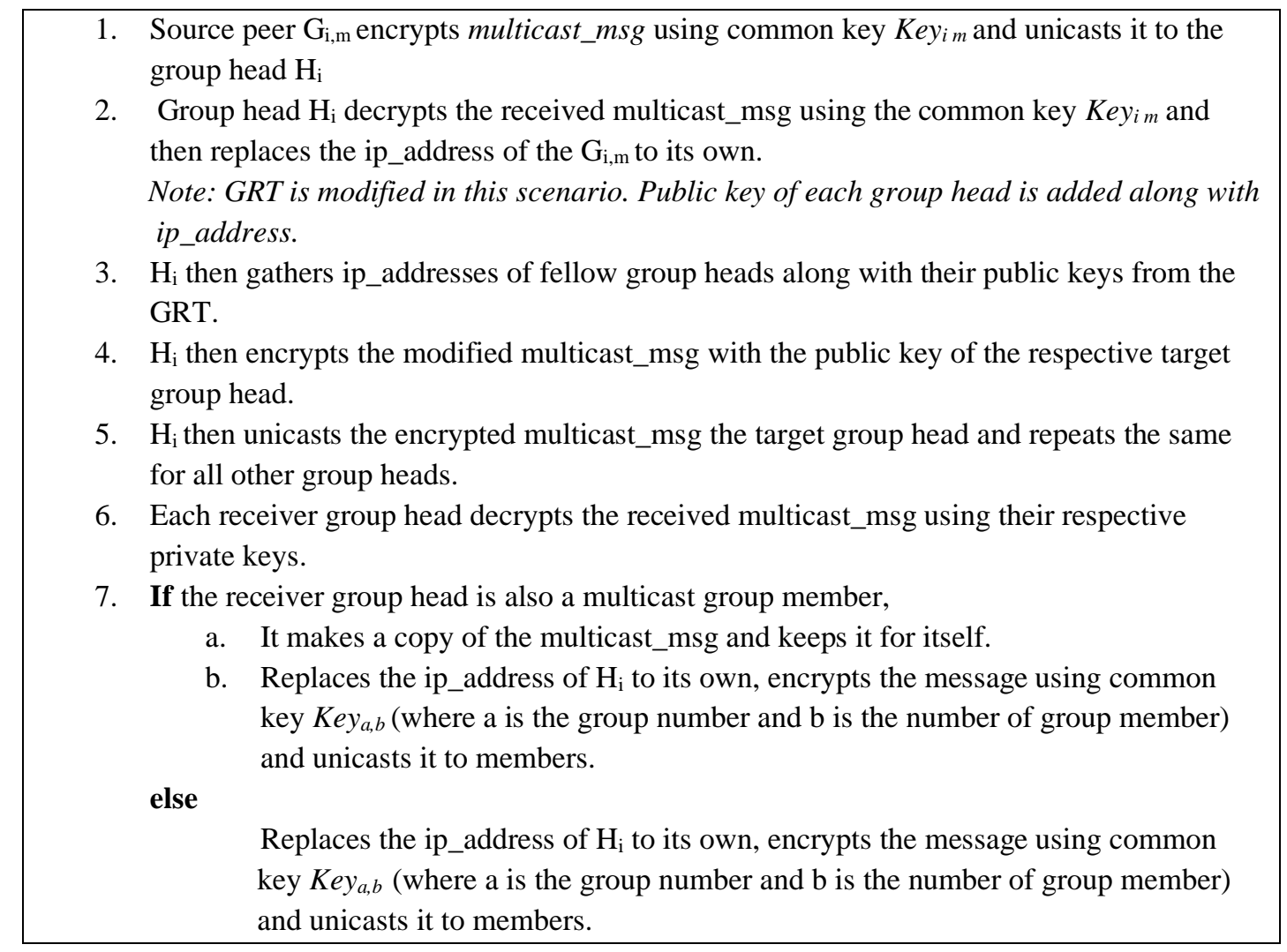

\section{Example 1:}

Scenario : $c_{s i} \geq n_{r}$ \# group heads $\left(\mathrm{n}_{\mathrm{r}}\right)=6$ Capacity of each group head $\left(c_{i}^{s_{i}}\right)=9$ In this example assume that the source peer is $\mathrm{G}_{4,18}^{\mathrm{s}}$
Fig. 5 Multicast Algorithm 1 with Security and Anonymity

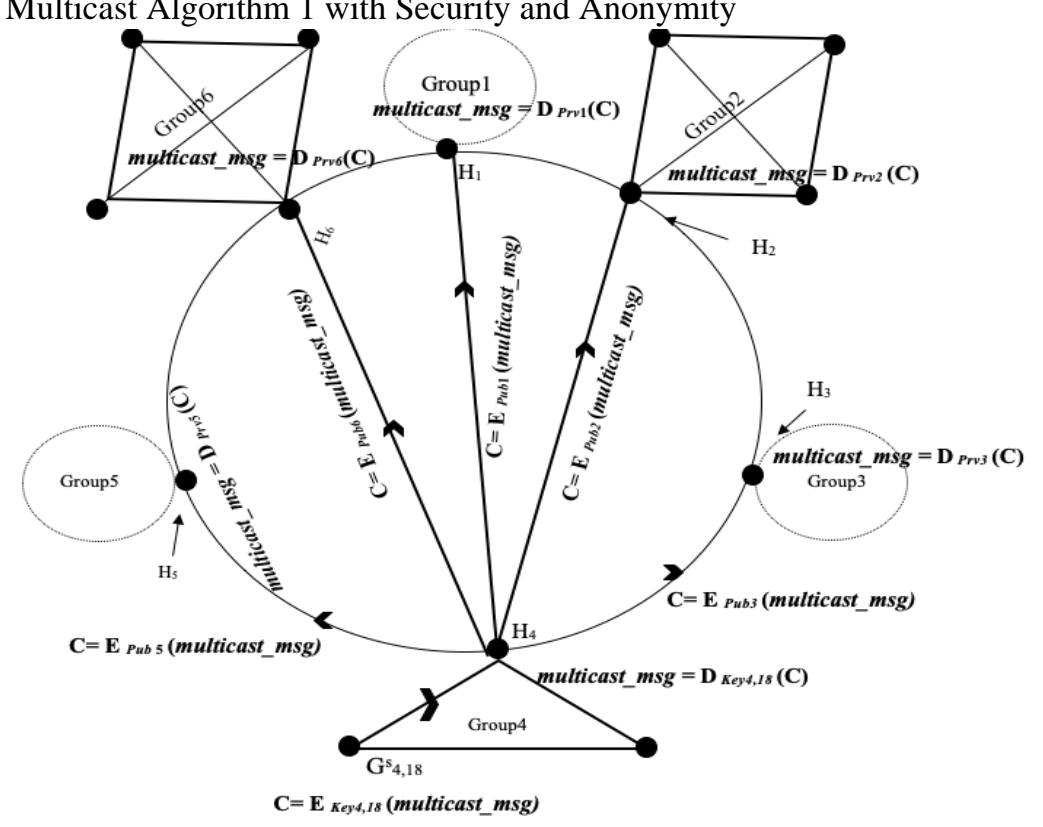

Fig. 6 Multicast Algorithm 1 example with Cryptographic messages 


\section{Secured Multicast Algorithm 2 with Anonymity where Capacity < \# groupheads}

1. Source peer $\mathrm{G}_{\mathrm{i}, \mathrm{m}}$ encrypts multicast_msg using common key Key ${ }_{i m}$ and unicasts it to the group head $\mathrm{H}_{\mathrm{i}}$

2. Group head $\mathrm{H}_{\mathrm{i}}$ decrypts the received multicast_msg using the common key Key $y_{i m}$ and then replaces the ip_address of the $\mathrm{G}_{\mathrm{i}, \mathrm{m}}$ to its own.

for $j=1$ to $p$

3. $\mathrm{H}_{\mathrm{i}}$ then gathers ip_addresses of fellow group heads along with their public keys from the GRT.

4. $\mathrm{H}_{\mathrm{i}}$ then encrypts the modified multicast_msg with the public key of the respective target group head $P u b_{a}$ where a is the number of the group head for each subset.

5. $\mathrm{H}_{\mathrm{i}}$ then unicasts the encrypted multicast_msg to the target group head and repeats the same for all other group heads $\mathrm{p}$ in $\mathrm{j}$.

6. Each receiver group head decrypts the received multicast_msg using private key Prva where $\mathrm{a}$ is the group head number.

7. If the receiver group head is also a multicast group member,

a. It makes a copy of the multicast_msg and keeps it for itself.

b. Replaces the ip_address of $\mathrm{H}_{\mathrm{i}}$ to its own, encrypts the message using common key $\operatorname{Key}_{a, b}$ (where a is the group number and $\mathrm{b}$ is the number of group member) and unicasts it to members.

else

Replaces the ip_address of $\mathrm{H}_{\mathrm{i}}$ to its own, encrypts the message using common key

$K e y_{a, b}$ (where $\mathrm{a}$ is the group number and $\mathrm{b}$ is the number of group member) and unicasts it to members.

Fig. 7 Multicast Algorithm 2 with Security and Anonymity

Example 2:

$\mathrm{c}_{\mathrm{si}}<\mathrm{n}_{\mathrm{r}}($ Case 1$)$

Suppose \#group heads $\left(\mathrm{n}_{\mathrm{r}}\right)=13$

Capacity of each group head $\left(\mathrm{c}^{\mathrm{s}} \mathrm{i}\right)=5$

In this example assume that the source peer is $\mathrm{G}^{\mathrm{s}}{ }_{7,18}$

So, Set T of 13 number of receiver group-heads is partitioned into 3 disjoint subsets.

Subset $\mathrm{T}_{1}$ consists of receiver groupheads $\mathrm{H}_{8}$ to $\mathrm{H}_{12}, \mathrm{~T}_{2}$ consists of receiver group-heads $\mathrm{H}_{13}, \mathrm{H}_{1}$ to $\mathrm{H}_{4}$ and subset $\mathrm{T}_{3}$ consists of receiver group-heads $\mathrm{H}_{5}$ and $\mathrm{H}_{7}$

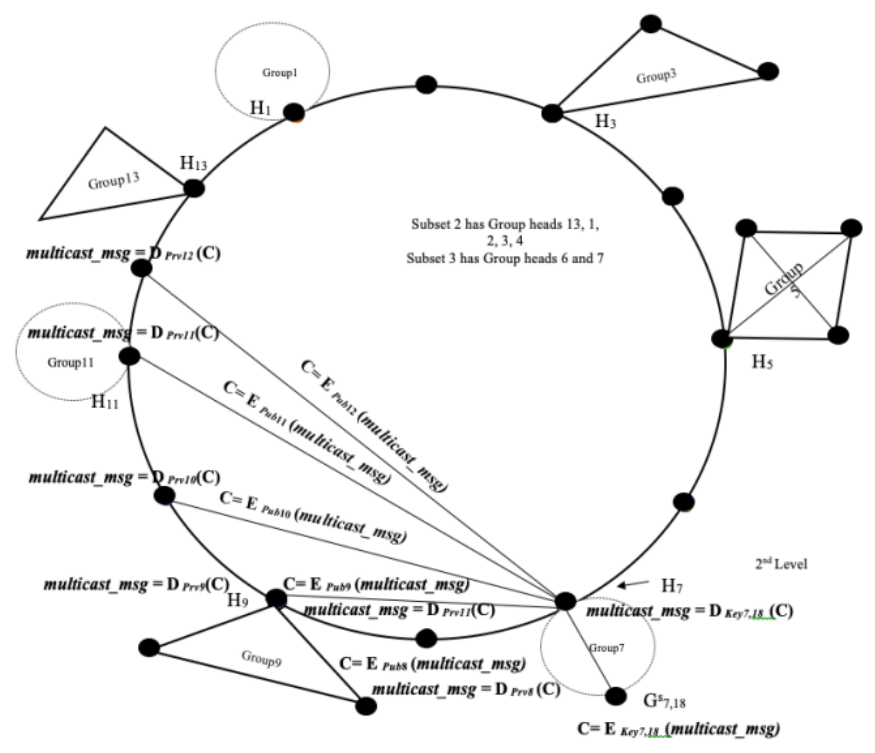

Fig. 8 Multicast Algorithm 2 example with Cryptographic messages 


\section{Secured Multicast Algorithm 3 with Anonymity where Capacity < \# groupheads}

1. Source peer $\mathrm{G}_{\mathrm{i}, \mathrm{m}}$ encrypts multicast_msg using common key Keyi $m$ and unicasts it to the group head $\mathrm{H}_{\mathrm{i}}$

2. Group head $\mathrm{H}_{\mathrm{i}}$ decrypts the received multicast_msg using the common key Key $m$ and then replaces the ip_address of the $\mathrm{H}_{\mathrm{i}, \mathrm{m}}$ with its own.

3. $\mathrm{H}_{\mathrm{i}}$ then gathers ip_addresses of fellow group heads along with their public keys from the GRT.

4. $\mathrm{H}_{\mathrm{i}}$ randomly selects groups heads equal to its capacity/degree.

5. It encrypts the modified multicast_msg with the public key of the selected group head Puba where a is the number of the group head and unicasts it.

6. $\mathrm{H}_{\mathrm{i}}$ repeats the same for all other selected group heads based on degree.

If receiver group head receives the multicast_msg for the first time (unique),

a. Each receiver group head decrypts the received multicast_msg using private key Prva.

If the receiver group head is also a multicast group member,

i. It makes a copy of the multicast_msg and keeps it for itself.

ii. Replaces the ip_address of $\mathrm{H}_{\mathrm{i}}$ to its own, encrypts the message using common key $K_{e} y_{a, b}$ (where $\mathrm{a}$ is the group number and $\mathrm{b}$ is the number of group member) and unicasts it to members.

else

i. Replaces the ip_address of $\mathrm{H}_{\mathrm{i}}$ to its own, encrypts the message using common key $K e y_{a, b}$ (where $\mathrm{a}$ is the group number and $\mathrm{b}$ is the number of group member) and unicasts it to members.

b. It then replaces the ip_address of $\mathrm{H}_{\mathrm{i}}$ to its own; acquires ip_address and public key of successor group head; encrypts the modified message using the acquired public key $P u b_{s}$ where $\mathrm{s}$ is the successor group head and forwards it.

c. Message propagation continues similarly in the $1^{\text {st }}$ level ring. else

Receiver group head drops the message.

Fig. 9 Multicast Algorithm 3 with Security and Anonymity

Example 3:

$\mathrm{c}_{\mathrm{si}}<\mathrm{n}_{\mathrm{r}}($ Case 2$)$

Suppose \#group heads $\left(\mathrm{n}_{\mathrm{r}}\right)=8$ Capacity of each group head $\left(\mathrm{c}_{\mathrm{i}}^{\mathrm{s}}\right)=3$

In this example assume that the source peer is $\mathrm{G}_{2,23}^{\mathrm{s}}$

In Fig $10, \mathrm{H}_{2}$ selects any 3 group heads in random (say $\mathrm{H}_{4}$, $\left.\mathrm{H}_{6}, \mathrm{H}_{7}\right)$

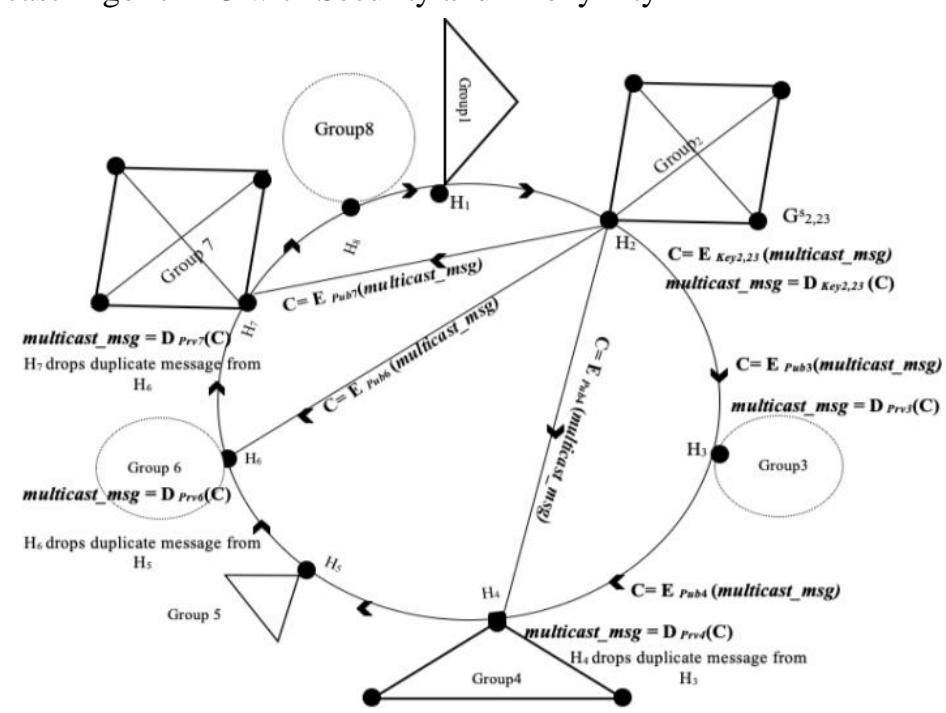

Fig. 10 Multicast Algorithm 3 example with Cryptographic messages 


\section{CONCLUSION}

In this paper, CRT based structured P2P architecture [9], [16] has been considered. We have designed secured data lookup protocols and secured multicast algorithms both inter and intra. In addition, we also considered the anonymity in case of multicasting.

\section{REFERENCES}

[1] Y.H. Chu, S. Rao, S. Seshan, and H. Zhang, “A Case for End System Multicast,” IEEE J. Selected Areas in Comm, vol. 20, no. 8, Oct. 2002.

[2] J. Jannotti, D. Gifford, K. Johnson, M. Kaashoek, and J. O’Toole, "Overcast: Reliable Multicasting with an Overlay Network," Proc. Symp. Operating Systems Design and Implementation (OSDI'00), Oct. 2000.

[3] C.K.S. Banerjee and B. Bhattacharjee, "Scalable Application Layer Multicast," Proc. ACM SIGCOMM'02, Aug. 2002.

[4] J. A. Dejan Kosti, A. Rodriguez, and A. Vahdat, "Bullet: High Bandwidth Data Dissemination Using an Overlay Mesh," Proc. Symp. Operating Systems Principles (SOSP,03), Oct. 2003.

[5] S. Banerjee, C. Kommareddy, B.B.K. Kar, and S. Khuller, "Construction of an Efficient Overlay Multicast Infrastructure for Real-Time Applications,” Proc. INFOCOM’03, Mar. 2003.

[6] A. Riabov and L. Z. Zhen Liu, "Overlay, Multicast Trees of Minimal Delay," Proc. Int'l. Conf. Distributed Computing Systems (ICDCS)'04), Mar. 2004.

[7] R. Zhang and Y. C. Hu, "Borg: A Hybrid Protocol for Scalable Application-Level Multicast in Peer-to-Peer Networks," Proc. Int'l. Workshop Network and Operating System Support for Digital Audio and Video (NOSSDAV'03), 2003.

[8] S. Ratnasamy, M. Handley, R. Karp, and S. Shenker, “Application Level Multicast Using ContentAddrerssable Networks,” Proc. Int'l. Workshop Networked Group Comm (NGC’01), 2001.

[9] Bidyut Gupta, Nick Rahimi, Henry Hexmoor, Shahram Rahimi, Koushik Maddali, and Gongzhu Hu, Design of Very Efficient Lookup Algorithms for a Low Diameter Hierarchical Structured Peerto-Peer Network, Proc. IEEE $16^{\text {th }}$ Int. Conf. Industrial Informatics (IEEE INDIN), July 2018, Porto, Portugal.

[10] Shiping Chen, Baile Shi, Shigang Chen, and Ye Xia, "ACOM: Any-Source Capacity-Constrained Overlay Multicast in Non-DHT P2P networks," IEEE Tran. Parallel and Distributed Systems, vol. 18, no. 9, pp. 1188-1201, Sep. 2007.

[11] N. Rahimi, K. Sinha, B. Gupta, and S. Rahimi, "LDEPTH: A low diameter hierarchical p2p network architecture," Proc. 2016 IEEE Int. Conf. on Industrial Informatics (IEEE INDIN), Poitiers, France, July, 2016.

[12] I. Stocia, R. Morris, D. Liben-Nowell, D. R. Karger, M. Kaashoek, F. Dabek, and H. Balakrishnan, "Chord: a scalable peer-to-peer lookup protocol for internet applications," IEEE/ACM Tran. Networking, vol. 11, No. 1, pp. 17-32, Feb. 2003.

[13] Stephen E. Deering and David R. Cheriton, "Multicast Routing in Datagram Internetworks and Extended LANs", ACM Trans. on Computer Systems (TOCS), Vol. 8, No. 2, pages. 85-110, May 1990.

[14] Tony A. Ballardie, “Core Based Tree Multicast Routing Architecture”, Internet Engineering Task Force (IETF), RFC 2201, September 1997.

[15] M. Yang and Y. Yang, "An Efficient Hybrid Peer-to-Peer System for Distributed Data Sharing", IEEE Trans. Computers, vol. 59, no. 9, pp. 1158-1171, Sep. 2010.

[16] Indranil Roy, Koushik Maddali, Swathi Kaluvakuri, Benafsheh Rekabdar, Ziping Liu, Bidyut Gupta and Narayan C. Debnath, Efficient Any Sourc Overlay Multicast in CRT- Based P2P Networks- A Capacity- Constrained Approach, Proc. IEEE 17 th Int. Conf. Industrial Informatics (IEEE INDIN), July 2019, Helsinki-Espoo, Finland. 